\title{
Fall Detection Using Commodity Smart Watch and Smart Phone
}

\author{
Ilias Maglogiannis ${ }^{1,2}$, Charalampos Ioannou ${ }^{3}$, \\ George Spyroglou, ${ }^{2,3}$, and Panayiotis Tsanakas ${ }^{3}$ \\ ${ }^{1}$ University of Piraeus, Dept. of Digital Systems, Piraeus, Greece \\ ${ }^{2}$ University of Thessaly, Dept. of Computer Science and Biomedical Informatics, \\ Lamia Greece \\ ${ }^{3}$ School of Electrical and Computer Engineering, National Technical University of Athens \\ imaglo@unipi.gr
}

\begin{abstract}
Human motion data captured from wearable devices such as smart watches can be utilized for activity recognition or emergency event detection, especially in the case of elderly or disabled people living independently in their homes. The output of such sensors is data streams that require real-time recognition, especially in emergency situations. This paper presents a novel application that utilizes the low-cost Pebble Smart Watch together with an Android device (i.e a smart phone) and allows the efficient transmission, storage and processing of motion data. The paper includes the details of the stream data capture and processing methodology, along with an initial evaluation of the achieved accuracy in detecting falls.
\end{abstract}

Keywords: Fall Detection, Smart watch, Activity Recognition, Ambient Assisted Living, Streaming data.

\section{Introduction}

The introduction of ubiquitous and mobile systems in human centered computing pushes towards achieving availability of invisible software applications and information anywhere and anytime [1]. Applications and interfaces able to automatically process data provided by sensors, exchange knowledge and make intelligent decisions in a given context, are strongly desirable. Natural user interactions with such applications are based on autonomy, avoiding the need for the user to control every action, and adaptivity, so that they are contextualized and personalized, delivering the right information and decision at the right moment. Thus, the development of assistive applications particularly for elderly or disabled people living on their own, has gained significant attention during the last years. The aging population is a significant issue. According to a recent report published by the EU the percentage of European population over the age of 65 is expected to be over $20 \%$ in 2020 and over $30 \%$ in 2060 [2].

The detection of falls is considered an essential function in the context of such intelligent assistive applications and environments, since approximately $33 \%$ of persons over the age of 65 and $50 \%$ of persons over the age of 85 experience a fall 
each year [3]-[4]. The injuries associated with falls can have serious consequences. For example, following hip fracture, $50 \%$ of older people are unable to live independently, 25\% will die within six months, and 33\% die within one year [3]-[4].

The goal of this work is to propose an unobtrusive wearable system capable of capturing and analyzing motion data and reaching a prompt decision for activity recognition and fall detection. For this purpose, the Pebble smart watch [5] has been utilized, as it combines several positive characteristics (low price, water-resistance, user friendliness, long battery life, open SDK). The specific smart watch has a reliable built-in accelerometer to measure human movements, and a personal area network interface in order to transmit data and communicate with other devices. More details about the Pebble device are provided in Section 2.2. The processing of the motion data occurs in a nearby Android device, since the smart watch lacks the necessary computing power to perform data analysis and the wide networking interface for transmitting the fall detection information. The overall architecture of the proposed fall detection application is illustrated in Figure 1.

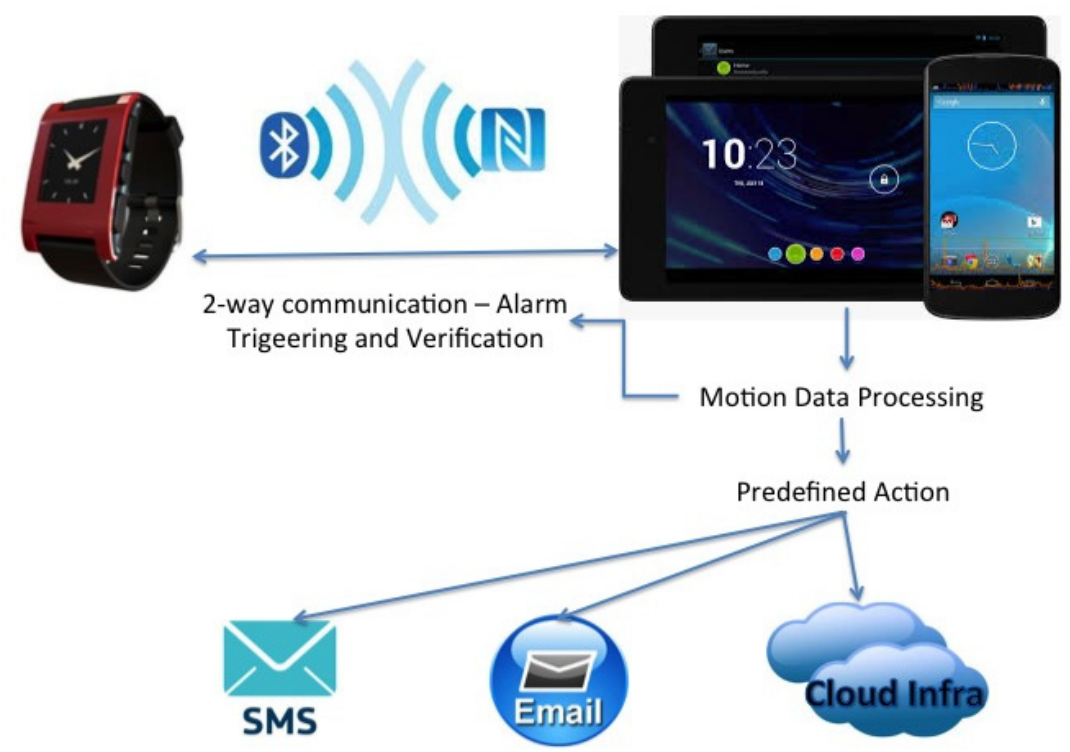

Fig. 1. Architecture of the proposed fall detection application

The rest of the paper is organized as follows: In Section 2, background material and related work is provided. Next, in Section 3 the SW of the proposed application that resides in both the smart watch and the smart phone are presented, while Section 4 is devoted to an initial evaluation. Finally, Section 5 covers concluding remarks and some future research directions. 


\section{Background Information and Related Work}

\subsection{Fall Detection Applications}

The concept of activity recognition with focus on fall detection is relatively new; however, it is quite popular due to the vast amount of sensors that have flooded the market recently. Several research groups and R\&D departments of prominent companies have developed similar applications and there already exists significant related research work in the field. A comprehensive review exists in [6]. Information regarding the human movement and activity in assisted environments is frequently acquired through visual tracking of the subject's or patient's position. Overhead tracking through cameras provides the movement trajectory of the patient and gives information about user activity on predetermined monitored areas [7]. A different approach for collecting patient activity information is the use of sensors that integrate devices like accelerometers, gyroscopes and contact sensors. The latter approach depends less on issues like patient physiology (e.g. body type and height) and environmental information (e.g. topology of monitored site) and can be used for a variety of techniques enabling user activity recognition [8-9]. In previous works [10-11] we have presented a patient fall detection system, based on such body sensors that utilized advanced classification techniques and Kalman filtering for producing post fall detection. In this work we are trying to exploit a new class of smart devices that has been introduced recently: smart watches. We have selected the Pebble smart watch as a popular representative and we present some details of its capabilities in the next section.

\subsection{The Pebble Smart Watch}

Smart-watches are wristwatches with a micro display, integrated sensors and connectivity. The intention of the manufactures of smart watches is to have a new device that displays short messages like SMS, RSS feeds or Facebook messages. The Pebble is a smart watch developed by Pebble Technology and released in 2013. It features a black and white e-paper display, a vibrating motor, a magnetometer, ambient light sensors and an accelerometer, enabling its use as an activity tracker. The Pebble is compatible with Android and iOS devices. When connected to a phone, it can receive a vibrating alert to text messages, emails, incoming calls, and notifications from social media accounts.

The watch has a 1.26 -inch $144 \times 168$ pixel black and white e-paper display using an ultra low-power "transflective LCD" with a backlight. The communication with an Android or iOS device is implemented using Bluetooth 4.0 (Bluetooth Low Energy) protocol. An important feature of the Pebble smart watch is that it is a solid and strong construction and it has a waterproof rating of $5 \mathrm{~atm}$, which means it can be submerged down to $40 \mathrm{~m}$. An open software development kit (SDK) is available to programmers for developing custom applications. The SDK (known also as PebbleKit) allows the two-way communication between Pebble and smartphones running iOS or Android via the AppMessage framework and includes access to the accelerometer, as well as a Javascript API. 


\section{The Proposed System}

As shown in Figure 1 (system architecture) and illustrated also in Figure 2, the proposed activity and fall detection system consists of two software modules, which work in conjunction and communicate with each other using the Bluetooth 4.0 networking interface. The first module runs on the Pebble smart watch, and it is responsible for the user interaction and the data input. The second module is executed on the android device and handles data processing and alarm management. In order the system to be fully functional, the user has to wear the Pebble watch with the fall detection application running and remains within the Bluetooth range of the android device. In case the Bluetooth device is a smart phone the user can carry the device, so it can be always within range; however, at a home setup, the android device could be a tablet, a board PC, or a set-top box.

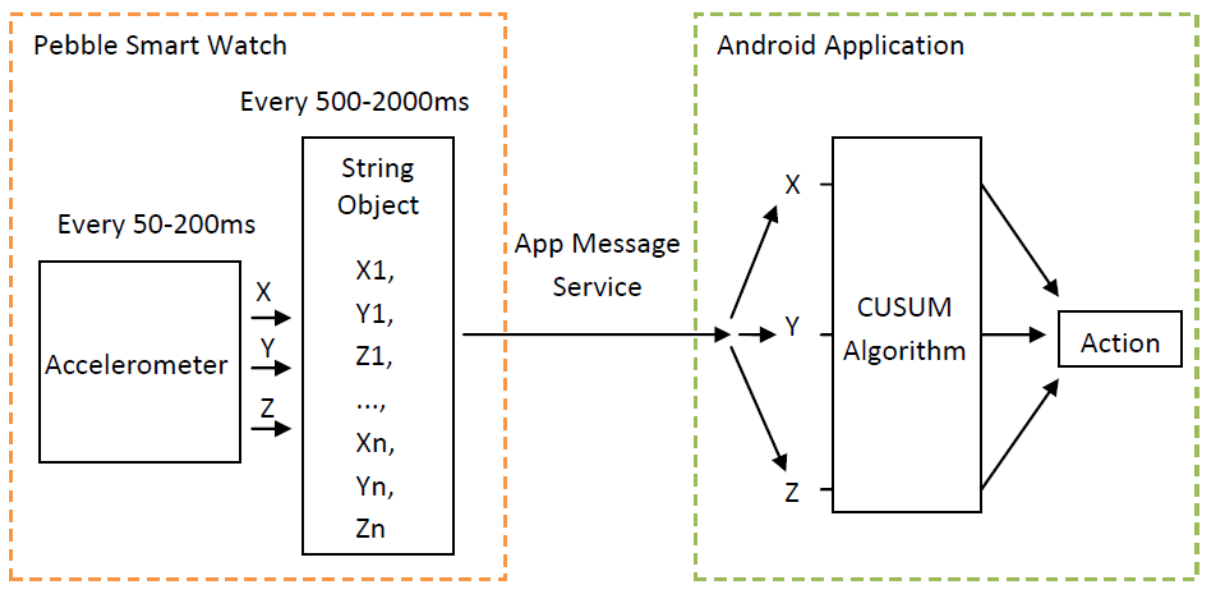

Fig. 2. The proposed fall detection application SW modules

\subsection{The Pebble Application}

The Pebble SDK 2.0 (until release 6), does not support multitasking, thus the user wearing the Pebble device should have the fall detection app installed in the foreground. Therefore, it is not feasible to run any other application on the background or to interact with other applications. The main screen of the developed app consists of a minimal clock, so the user can be informed about the time. Also at the bottom of the main screen, there is a small text field, which displays the status of the connection with the android device. If the android device is connected with the Pebble, the text field displays "Connected - Fall Detection is Active", whereas if the Pebble is not connected, the text field displays "Disconnected - Fall Detection is not Active" (see Figure 3).

As the Pebble display shows the time, the developed application checks whether an active Bluetooth (BT) connection with the android device is established. If a connection is found, the smart watch transmits the current accelerometer data and the corresponding time stamps. In the case for some reason the Pebble smart watch is disconnected from the 
android device (for instance, it gets out the BT range), the Pebble application changes the visible status connection, closes the accelerometer, stops transmitting data and notifies the user with a small vibration about the change in the status of the connection. The user has to be notified immediately about the disconnection. Once reconnected, the application starts again automatically.

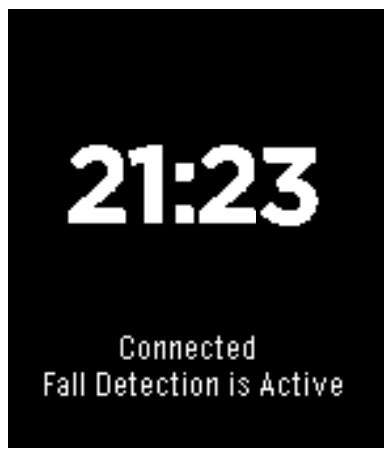

Fall Detection

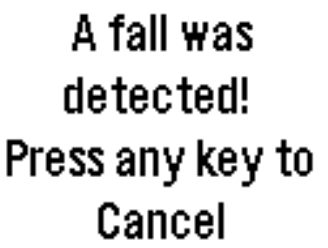

Fig. 3. Screenshots of the Pebble Smart watch Application in case of an idle active connection and in case of a detected fall

The code that runs on the Pebble watch is written in the $\mathrm{C}$ programming language. A very effective feature of the SDK is the "event handler" that supports the triggering of various functions, according to the captured event. The smart watch is capable of receiving 3-axis integer (example: $\mathrm{x} 1, \mathrm{y} 1, \mathrm{z} 1$ ) motion data from the built-in accelerometer in a sampling period of $50 \mathrm{~ms}$, and stores them into character array variables, separated by commas. The values are converted into character array, are then separated by commas and copied to a single character array, by using the using the standard $\mathrm{C}$ function, strcat(). The final string is as follows, for ten samples or 500ms: $[\mathrm{x} 1, \mathrm{y} 1, \mathrm{z} 1, \mathrm{x} 2, \mathrm{y} 2, \mathrm{z} 2, \mathrm{x} 3, \mathrm{y} 3, \mathrm{z} 3, \mathrm{x} 4, \mathrm{y} 4, \mathrm{z} 4, \mathrm{x} 5, \mathrm{y} 5, \mathrm{z} 5, \mathrm{x} 6, \mathrm{y} 6, \mathrm{z6}, \mathrm{x} 7, \mathrm{y} 7, \mathrm{z} 7, \mathrm{x} 8, \mathrm{y} 8, \mathrm{z} 8$, $\mathrm{x} 9, \mathrm{y} 9, \mathrm{z} 9, \mathrm{x} 10, \mathrm{y} 10, \mathrm{z} 10]$.

Every 500ms the Pebble converts the character array into a tuplet string dictionary, by using a characterization key. After the serialization procedure, Pebble sends the data as a single string via the BT interface, while at the same time it continues to receive and store the incoming accelerometer data. The data is then sent to the android device for further processing, according to fall detection algorithms. In case a fall is detected, the android device sends a string value (fall-alarm) to the Pebble, and the proper event handler changes the watch status from watch view and status view, into alarm view. Alarm view consists of a text field, which displays: "A fall was detected Press any key to Cancel", the Pebble screen's backlight is active and a vibration is triggered every $500 \mathrm{~ms}$.

If the user presses any of the Pebble's buttons in 5 seconds after the fall is detected, the fall alarm will be set off and a cancel-alarm string value will be sent to the android device. If the user does not respond within the 5-second interval the Pebble will send a trigger-alert sting value with the proper event handler, to notify about the triggered alarm. 


\subsection{The Android Application}

The Android application has been developed in Java using the Eclipse Android Developer Tools, intended for android devices. This app is launched (in background mode), every time the user launches the app on the Pebble smart watch and vise versa.

When the app is active, whether it runs on background or on foreground, it receives data from the Pebble device. The incoming string using the same tuplet dictionary and characterization key, is converted into comma-separated integer numbers.

According to the corresponding accelerometer axis, the data is parsed into the fall detection algorithm as follows: $\mathrm{X}=[\mathrm{x} 1, \mathrm{x} 2, \mathrm{x} 3, \mathrm{x} 4, \mathrm{x} 5, \mathrm{x} 6, \mathrm{x} 7, \mathrm{x} 8, \mathrm{x} 9, \mathrm{x} 10], \mathrm{Y}=[\mathrm{y} 1, \mathrm{y} 2, \mathrm{y} 3, \mathrm{y} 4$, $\mathrm{y} 5, \mathrm{y} 6, \mathrm{y} 7, \mathrm{y} 8, \mathrm{y} 9, \mathrm{y} 10], \mathrm{z}=[\mathrm{z} 1, \mathrm{z} 2, \mathrm{z} 3, \mathrm{z} 4, \mathrm{z} 5, \mathrm{z} 6, \mathrm{z} 7, \mathrm{z} 8, \mathrm{z} 9, \mathrm{z} 10]$. The implemented fall detection algorithm has been proposed in a previous work and is based on the cumulative sum (CUSUM) change detection algorithm [12]. The CUSUM decision function, as calculated for a fall in the X-and Y-axis, is depicted in Figure 4. In case a fall is detected a fall-alarm string will be sent to the Pebble and as already mentioned if the user does not cancel the alarm within the appropriate time interval, the application will initiate an actual alarm, transmitting automatically the fall detection information as defined (i.e. via email or via a call or SMS in the case of a smart phone).

\section{The System in Practice - Initial Experimental Results}

The main goal of this work is to present a user-friendly and unobtrusive application for fall detection. The proposed system combines the advantages of two simple, lowcost, and widely available devices. The obvious advantages of the smart watch are the ease and comfort of use, the high quality of acquired sensor data, the minimum weight and the low power consumption characteristics. The limited computing resources are replaced by the processing power of a typical android smartphone. The proposed solution is based on the bare minimum sampling rate (i.e. 50-200 ms), in order to minimize power consumption and increase battery life. During our experiments, the Pebble smart watch was proven capable of continuous sampling for at least 30 hours. An improvement in autonomy was achieved taking into consideration the actual time and the frequency of the user's movement, in order to decrease even more the sampling rate during the sleep. That resulted in a combined 35+ hours of continuous user fall tracking with a single battery charging. 

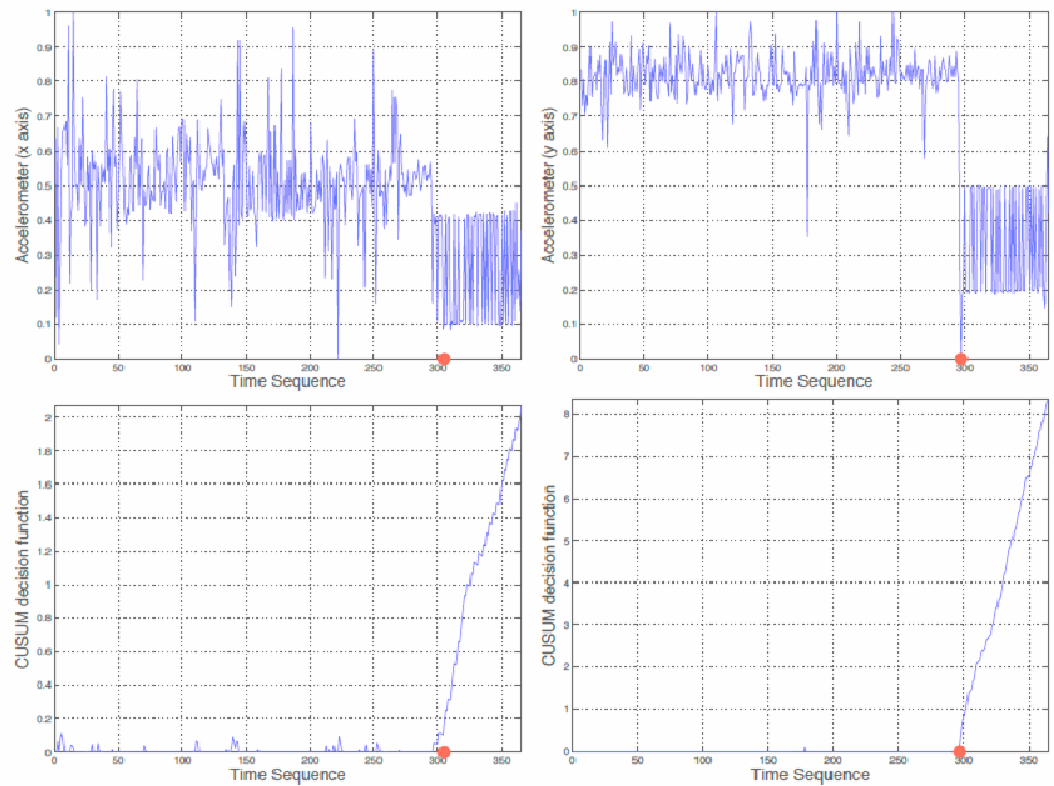

Fig. 4. Accelerometer readings and the corresponding CUSUM calculation in case of a typical fall

A significant parameter of the CUSUM algorithm presented in [12] is the value of the threshold for the CUSUM decision function, which determines a fall. This is symbolized as the $\mathrm{h}$ parameter in the specific algorithm. According to the performed experiments, a lower value for $\mathrm{h}$ corresponds to smaller delays in fall detection, but produces more false positives. By experimenting with the h-parameter, we tried to minimize false positive alarms, ensuring the minimum possible delay time. The results of the conducted experiments are presented in Table 1.

Table 1. Number of false alarms and the delay for several values of $h$, for each axis

\begin{tabular}{|l|l|l|l|l|l|l|l|}
\hline \multicolumn{1}{|c|}{$\mathrm{h}$} & 2.5 & 3.0 & 3.4 & 3.6 & 3.8 & 4.0 & 5.0 \\
\hline \multicolumn{7}{|c|}{ Accelerometer (X-axis) } \\
\hline False Alarms & 5 & 3 & 2 & 1 & 0 & 0 & 0 \\
\hline Delay (ms) & 0 & 16 & 46 & 43 & 10 & 10 & 10 \\
\hline \multicolumn{7}{|c|}{ Accelerometer (Y-axis) } \\
\hline False Alarms & 7 & 5 & 4 & 3 & 3 & 2 & 0 \\
\hline Delay (ms) & 1 & 0 & 10 & 16 & 45 & 18 & 10 \\
\hline \multicolumn{7}{|c|}{ Accelerometer (Z-axis) } \\
\hline False Alarms & 8 & 6 & 5 & 4 & 4 & 3 & 0 \\
\hline Delay (ms) & 14 & 18 & 31 & 10 & 40 & 45 & 10 \\
\hline
\end{tabular}




\section{$5 \quad$ Discussion and Conclusions}

This paper presents a fall detection application based on the Pebble smart watch, which is capable of transmitting the built-in accelerometer readings via Bluetooth to an android device for further processing. To the best of our knowledge, this is the first integrated application developed for fall detection based on a commodity smart watch. The main advantages of the proposed application are that it is non-obtrusive, low-cost, easy to deploy and use, and suitable for processing streaming data and reaching a prompt decision. In a future work, we intend to combine contextual data of the android device (e.g. location, audio, etc.), in order to decrease false alarms and improve the accuracy of the system and add additional functionalities, such as fall severity estimation. Furthermore the same device will be used for the simultaneously assessing sleep and other vital activities.

Acknowledgment. The authors would like to thank the European Union (European Social Fund ESF) and Greek national funds for financially supporting this work through the Operational Program "Education and Lifelong Learning" of the National Strategic Reference Framework (NSRF) - Research Funding Program: \Thalis \Interdisciplinary Research in Affective Computing for Biological Activity Recognition in Assistive Environments. Part of the work reported here has been carried out in the framework of national project Providing Integrated eHealth Services for Personalized Medicine utilizing Cloud Infrastructure (PinCloud), led by the University of Piraeus, conducted in the context of the National Strategic Reference Framework NSRF 20072013, Cooperation 2011 and co-funded by the European Commission.

\section{References}

1. Varshney, U.: Pervasive Healthcare. IEEE Computer Magazine 36(12), 138-140 (2003)

2. 2012 Ageing Report: Economic and budgetary projections for the 27 EU Member States (2010-2060)

3. West, J., Hippisley-Cox, J., Coupland, C., Price, G., Groom, L., Kendrick, D., Webber, E.: Do rates of hospital admission for falls and hip fracture in elderly people vary by socioeconomic status? Public Health 118(8), 576-581 (2004)

4. Robinson, B., Gordon, J., Wallentine, S., Visio, M.: Relationship between lower-extremity joint torque and the risk for falls in a group of community dwelling older adults. Physiotherapy Theory and Practice 20, 155-173 (2004)

5. Official Pebble Web Site, https: / / getpebble.com

6. Mubashir, M., Shao, L., Seed, L.: A survey on fall detection: Principles and approaches. Neurocomputing 100, 144-152 (2013)

7. Doukas, C.N., Maglogiannis, I.: Emergency fall incidents detection in assisted living environments utilizing motion, sound, and visual perceptual components. IEEE Transactions on Information Technology in Biomedicine 15(2), art. no. 5623343, 277-289 (2011)

8. Zhang, Z., Kapoor, U., Narayanan, M., Lovell, N.H., Redmond, S.J.: Design of an unobtrusive wireless sensor network for nighttime falls detection. Proceedings of the Annual International Conference of the IEEE Engineering in Medicine and Biology Society, EMBS, art. no. 6091305, 5275-5278 (2011) 
9. Noury, N., Herve, T., Rialle, V., Virone, G., Mercier, E., Morey, G., Moro, A., Porcheron, T.: Monitoring behavior in home using a smart fall sensor and position sensors. In: Proceedings of 1st Annual International Conference on Microtechnologies in Medicine and Biology, pp. 607-610 (2000)

10. Doukas, C., Maglogiannis, I.: Advanced classification and rules based evaluation of motion, visual and biosignal data for patient fall incident detection. International Journal on Artificial Intelligence Tools, 175-191 (2010)

11. Doukas, C., Maglogiannis An, I.: assistive environment for improving human safety utilizing advanced sound and motion data classification. Universal Access in the Information Society 10(2), 217-228 (2011)

12. Tasoulis, S.K., Doukas, C.N., Maglogiannis, I., Plagianakos, V.P.: Statistical data mining of streaming motion data for fall detection in assistive environments. In: Annual International Conference of the IEEE Engineering in Medicine and Biology Society (2011) 